Classification

Physics Abstracts

68.15

\title{
Theory of the dynamics of spreading of liquids on fibers
}

\author{
Françoise Brochard-Wyart $\left({ }^{1}\right)$, Jean-Marc di Meglio $\left({ }^{2}\right)$ and David Quéré $\left({ }^{2}\right)$ \\ ( $\left.{ }^{1}\right)$ Laboratoire Structure et Réactivité aux Interfaces $\left({ }^{*}\right)$, Université Pierre et Marie Curie, 4 \\ place Jussieu, 75252 Paris Cedex 05, France \\ $\left({ }^{2}\right)$ Laboratoire de Physique de la Matière Condensée $\left({ }^{* *}\right)$, Collège de France, 11 place Marcelin \\ Berthelot, 75231 Paris Cedex 05, France
}

(Reçu le 12 juillet 1989, révisé le 19 octobre 1989, accepté le 26 octobre 1989)

\begin{abstract}
Résumé. - Nous étudions théoriquement l'étalement d'un liquide non volatil sur un cylindre solide. Après avoir établi comment se formait un ménisque macroscopique, nous nous attachons plus particulièrement à la dynamique de la formation de films microscopiques dus aux interactions de longue portée de type van der Waals.

Abstract. - We present a theoretical investigation of the spreading of a non-volatile liquid on a solid cylinder. We first derive the laws for the dynamics of the formation of the meniscus and we then focus particularly on the growth of microscopic films due to long range interactions between solid and liquid.
\end{abstract}

\section{Introduction.}

It is impossible to coat a fiber with a macroscopic film of liquid : the cylindrical coating breaks into a periodic array of droplets in order to minimize its interfacial energy. This is the well known Rayleigh instability [1].

If long range forces are taken into account, it becomes possible to stabilize films on fibers [2] (or in capillary tubes [3]) when these films are very thin (usually in the $100 \AA$ range). The statics of wetting of curved surfaces has been well described, both experimentally and theoretically $[2,4-6]$. The aim of this paper is to understand the dynamics of spontaneous spreading of a wetting, non-volatile liquid in two different situations :

i) the spreading of a very large drop on an horizontal cylinder ;

ii) the ascension of a liquid onto a vertical cylinder from a reservoir. We have already experimentally studied this situation [7].

This study is limited to the case of «dry wetting " [8] : we shall assume throughout this paper that the liquid is non-volatile and thus is not in equilibrium with its vapor, which allows us to consider spreading parameters larger than zero.

$\left({ }^{*}\right)$ UA $1319 \mathrm{du}$ CNRS.

$(* *)$ UA $792 \mathrm{du}$ CNRS. 
We shall give numerical estimates for all quantities which can be measured.

We first review some main results on the statics of wetting.

\section{Statics.}

2.1 SPREADING ON A PLANAR SURFACE. - The main parameter which determines the evolution of a non-volatile liquid deposited on a solid is the spreading parameter $S=\gamma_{\mathrm{SV}}-\gamma_{\mathrm{SL}}-\gamma$, where $\gamma_{\mathrm{SV}}$ is the solid/vacuum (or air) interfacial tension, $\gamma_{\mathrm{SL}}$ is the solid/liquid one and $\gamma$ is the interfacial tension between liquid and vacuum (or air). Depending on the sign of $S$, the drop will spread out spontaneously $(S>0)$ or only partially wet a surface $(S<0)$, in which case it has a finite contact angle $\theta_{\mathrm{e}}$ given by the Young equation :

$$
\cos \theta_{\mathrm{e}}=1+\frac{S}{\gamma}
$$

For $S \geqslant 0$, the liquid spreads out and forms a thin film on the solid: the long range interactions then need to be taken into account. For non retarded van der Waals interactions, the energy $W(e)$ per unit area of a film of thickness $e$ is :

$$
W(e)=-\frac{A_{\mathrm{SLV}}}{12 \pi e^{2}}
$$

$A_{\mathrm{SLV}}$ is the effective Hamaker constant describing the interaction of solid (S) and vacuum (V) (or air) separated by liquid (L). For perfect wetting conditions, $A_{\mathrm{SLV}}$ is negative and a molecular length a can be introduced for convenience [8] :

$$
a=\left(-\frac{A_{\mathrm{SLV}}}{6 \pi \gamma}\right)^{1 / 2}
$$

We estimate $a$ to be $3 \AA$ in what follows (this value is typical of a polymeric fiber/organic solvent system).

The energy $f$ of a thin film of area $\mathcal{A}$ is :

$$
f=f_{0}-S \mathcal{A}+W(e) \mathcal{A} .
$$

Minimizing this energy while keeping the volume $A e$ constant yields :

$$
S=W(e)+e \Pi(e)
$$

where $\Pi(e)$ is the pressure associated with the long range energy :

$$
\Pi(e)=-\frac{\mathrm{d} W(e)}{\mathrm{d} e}=\frac{\gamma a^{2}}{e^{3}},
$$

and is the so-called disjoining pressure introduced by Derjaguin [9]. The effect of such long range forces is to thicken the film (since $W(e) \rightarrow 0$ when $e \rightarrow \infty$ ).

For $S>0$, the thickness of the film is [10] :

$$
e_{\mathrm{s}}=a \sqrt{\frac{3 \gamma}{2 S}} \quad\left(e_{\mathrm{s}} \simeq 12 \AA \text { for } \frac{S}{\gamma}=0.1\right) .
$$


2.2 SPREADING ON A CYLINDER : SHEATH-DROP TRANSITION [2]. - For fibers, the situation is quite different because of the curvature of the interfaces. Let us compare the two following situations : a liquid droplet of volume $\Omega$ on a fiber of radius $b$ (Fig. 1a) and a liquid sheath of the same volume and of thickness $e(e \ll b)$ (Fig. 1b). The energy per unit volume $f_{\mathrm{d}}$ of the drop is dominated by the surface energy of the free liquid surface :

$$
f_{\mathrm{d}} \simeq \frac{\gamma}{\Omega^{1 / 3}} \simeq 0 \text { for a large drop }\left(\Omega^{1 / 3} \gg b\right)
$$

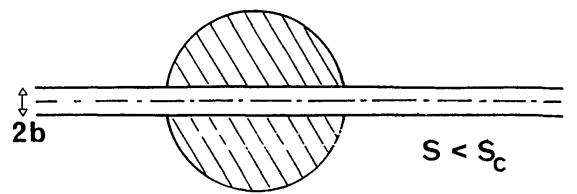

a)

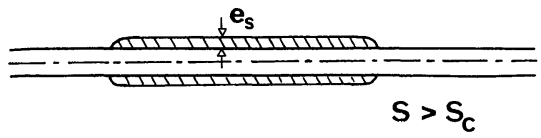

b)

Fig. 1. - a) Unspread drop on a fiber. b) Spread film on a fiber.

The free energy $f_{\mathrm{f}}$ of the liquid film of length $L$ is :

$$
f_{\mathrm{f}}=\frac{1}{\Omega} 2 \pi b L\left(-S+W(e)+\gamma \frac{e}{b}\right)
$$

where $\Omega=\pi L\left(e^{2}+2 e b\right)$ and where the wetted surface area is $2 \pi b L$. The first two terms also show the competition between $S$ (which tends to spread the film) and $W(e)$ (whose effect is to thicken the film); the third term is due to the effect of curvature since the outside surface (liquid/air interface) is larger than the inner one (solid/liquid interface). The energy difference $f\left(f=f_{\mathrm{f}}-f_{\mathrm{d}}\right)$ as a function of $e$ is pictured in figure 2. For $S$ larger than a threshold $S_{\mathrm{c}}, f$ can be negative. The droplet then spreads out and forms a liquid sheath of thickness $e$ given by the minimum of $\mathrm{f}$. The threshold is given by $f=0$ and $\frac{\partial f}{\partial e}=0$, with the result :

$$
S_{\mathrm{c}}=\frac{3}{2} \gamma\left(\frac{a}{b}\right)^{2 / 3}
$$

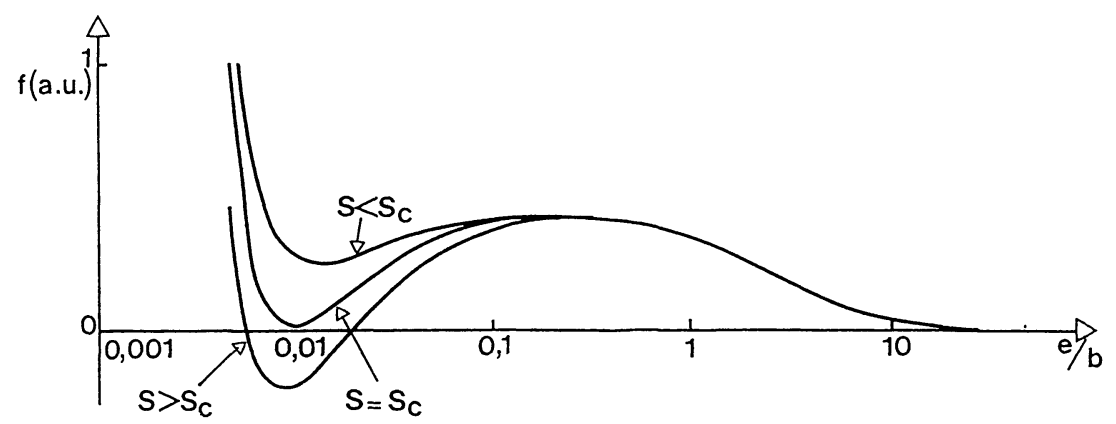

Fig. 2. - Energy difference (per unit volume) between drop and film for different spreading parameters. 
where $S_{\mathrm{c}}$ is strictly positive. The spreading transition occurs for $S \geqslant S_{\mathrm{c}}$ instead of $S \geqslant 0$, as was the case for a planar surface.

$$
\left(\frac{S_{\mathrm{c}}}{\gamma} \simeq 10^{-3} \text { for } b=10 \mu \mathrm{m}\right)
$$

and the thickness of the sheath is $e_{\mathrm{c}}=a^{2 / 3} b^{1 / 3}\left(e_{\mathrm{c}} \simeq 100 \AA\right)$.

For $S>S_{\mathrm{c}}$, the minimization yields :

$$
e=e_{\mathrm{s}}=a \sqrt{\frac{3 \gamma}{2 S}} \quad\left(e_{\mathrm{s}} \simeq 12 \AA\right) .
$$

This expression for $e$ is the same as for planar surfaces. This latter thickness implies very small amounts of liquid and so usually a « reservoir » drop will coexist with a wetting film, the thickness of which can be simply determined from the equality of the pressures inside the drop (of radius $R$ ) $P_{\mathrm{d}}$ and inside the film $P_{\mathrm{f}}$ :

$$
\begin{aligned}
& P_{\mathrm{d}} \simeq \frac{2 \gamma}{R} \simeq 0 \\
& P_{\mathrm{f}}=\frac{\gamma}{b+e}-\Pi(e) \simeq \frac{\gamma}{b}-\Pi(e) \quad(\text { for } e \ll b)
\end{aligned}
$$

which gives a thickness of :

$$
e=e_{\mathrm{c}}=a^{2 / 3} b^{1 / 3}
$$

for the film in equilibrium with a reservoir.

2.3 ASCENSION OF A LIQUid ALONG A CYLINDER (Fig. 3). - Now we assume that the fiber is vertical and is partially dipped in a large reservoir of liquid. At the bottom of the fiber there is a macroscopic meniscus, and if $S \geqslant S_{\mathrm{c}}$ a microscopic film will develop. The thickness $e$ of the liquid is now a function of $h$, the height above the reservoir.

The free energy of the system is :

$$
f=2 \pi \int \mathrm{d} h\left\{\gamma \sqrt{1+\left(\frac{\mathrm{d} e}{\mathrm{~d} h}\right)^{2}}(b+e)+b\left(\gamma_{\mathrm{SL}}-\gamma_{\mathrm{SO}}\right)+b W(e)+\rho g h e\left(b+\frac{e}{2}\right)\right\} .
$$

The first two terms are associated with interfacial energies, the third one with the long-range interactions and the last one with gravity ( $\rho$ is the density of the liquid, and $g$ the gravitational acceleration). This is a general equation and describes two regimes: the macroscopic meniscus and, in case of spreading $\left(S \geqslant S_{\mathrm{c}}\right)$, the microscopic film above the meniscus.

- Meniscus. - The long range term is negligible in (15) and we restrict attention to the case of very thin fibers whose radius $b$ is assumed to be much smaller than the capillary length $\kappa^{-1}$ :

$$
\kappa^{-1}=\sqrt{\frac{\gamma}{\rho g}} \quad\left(\kappa^{-1} \simeq 0.1 \mathrm{~cm}\right)
$$

and therefore gravity can also be neglected. By minimizing (15), we obtain :

$$
2 \pi(b+e) \gamma \cos \theta=2 \pi b(S+\gamma)
$$




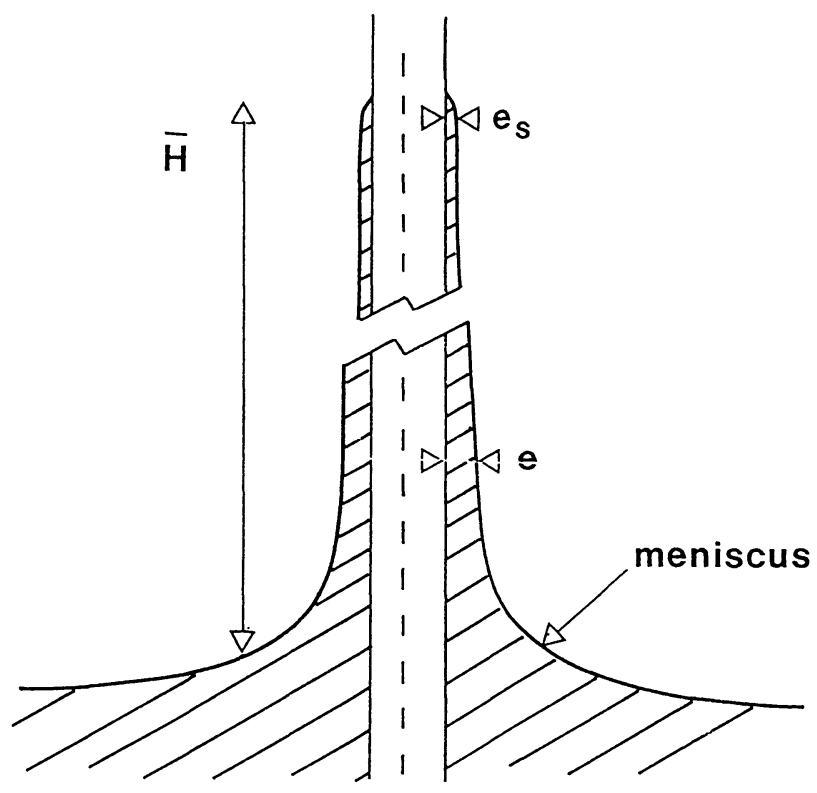

Fig. 3. - Equilibrium state of the « vertical spreading» situation.

which describes the equilibrium at the liquid surface between the interfacial tensions. This equation can be solved exactly $\left(\operatorname{tg} \theta=\frac{\mathrm{d} e}{\mathrm{~d} h}\right)$ and it is found that the meniscus adopts a catenary profile described by

$$
e(h)=\left(1+\frac{S}{\gamma}\right) b \cosh \left(\frac{h-h_{0}}{b\left(1+\frac{S}{\gamma}\right)}\right)-b
$$

where $h_{0}$ is an integration constant. For $S=0$ (i.e. a zero contact angle between solid and liquid), the meniscus climbs to a height $h_{0}$ with zero thickness. Notice that this profile implies that the liquid surface is perturbed at an infinite distance from the fiber, a result for having neglected the effects of gravity. We can estimate $h_{0}$ by introducing a higher cut-off for the thickness $e$. If we assume that the meniscus has a maximum thickness of $\kappa^{-1}$, we find :

$$
h_{0}=b \cosh ^{-1}\left(\frac{\kappa^{-1}}{b}\right) \simeq b \log \frac{2 \kappa^{-1}}{b} .
$$

The height $h_{0}$ is then equal to the radius of the fiber times a factor of order 6 . The profile of the meniscus (18) can also be obtained from the Laplace equation which says that the total curvature of the meniscus is equal to zero if gravity is neglected.

For $S=S_{\mathrm{c}}$, the thickness at $h=h_{0}$ is $\frac{3}{2} e_{\mathrm{c}}\left(e_{\mathrm{c}}\right.$ is given by (14)) and the meniscus ends up with a microscopic film.

For negative $S$, the meniscus ends up with a contact angle $\theta_{\mathrm{e}}$ at height $h_{\mathrm{m}}$ defined by :

$$
h_{\mathrm{m}}=h_{0}-b \cos \theta_{\mathrm{e}} \cosh ^{-1}\left(\frac{1}{\cos \theta_{\mathrm{e}}}\right) \text {. }
$$


- Microscopic film [2] $\left(S \geqslant S_{\mathrm{c}}\right)$. - Above the meniscus, a microscopic film should spread spontaneously.

In this case, the curvature $\left(\frac{\mathrm{d} e}{\mathrm{~d} h}\right)^{2} \ll 1$ and we assume again that $e \ll b$. For $S>S_{\mathrm{c}}$, we have seen that for a horizontal fiber, the thickness of the static film was $e=a^{2 / 3} b^{1 / 3}$. If the fiber is vertical, the thickness of the microscopic film will depend on the height $h$ above the reservoir, and the profile can be easily deduced from the pressure equilibrium condition :

$$
-\rho g h=\frac{\gamma}{b}-\Pi(e)
$$

and :

$$
e(h)=\frac{a^{2 / 3} b^{1 / 3}}{\left(1+\frac{h b}{\kappa^{-2}}\right)^{1 / 3}}
$$

Gravity has no influence for $h<\frac{\kappa^{-2}}{b}$. The thickness slowly decreases from $e=e_{\mathrm{c}}=a^{2 / 3} b^{1 / 3}$ ( $h=0$ in the above equation) to $e=e_{\mathrm{s}}$ (minimum thickness) for $h=\bar{H}$ :

$$
\bar{H}=\frac{\kappa^{-2}}{a}\left(\frac{2 S}{3 \gamma}\right)^{3 / 2}-\frac{\kappa^{-2}}{b} .
$$

Substituting for typical values, we find $\bar{H} \simeq 60 \mathrm{~m}$, a very long height indeed.

- Crossover between meniscus and film $\left(S \geqslant S_{\mathrm{c}}\right)$. - In the crossover region, we can neglect gravity as $h_{0} \ll \frac{\kappa^{-2}}{b}$. After minimization of (15), one integration gives :

$$
\frac{1}{2} \gamma\left(\frac{\partial e}{\partial h}\right)^{2}(b+e)-\gamma e+b S-b W(e)=0 .
$$

We can use this equation to determine the angle $\theta \simeq \frac{\partial e}{\partial h}$, for $S=S_{\mathrm{c}}$ in the crossover region where $h \simeq h_{0}$ and $e \simeq \frac{3}{2} e_{\mathrm{c}}$. We obtain :

$$
\theta \simeq \frac{2}{3}\left(\frac{e_{\mathrm{c}}}{b}\right)^{1 / 2}=\frac{2}{3}\left(\frac{a}{b}\right)^{1 / 3} \quad\left(\theta \simeq 10^{-2}\right) .
$$

2.4 STABILITY OF WETTING FILMS [11]. - As stated in the introduction, it is well known that cylindrical films are usually unstable to any axisymmetrical fluctuations and will break into an array of droplets [1]. Nevertheless, microscopic films do exist because they are stabilized by van der Waals interactions. It is easy to compute the threshold value $e_{0}$ above which the film will break : if $P_{\mathrm{f}}$ is the pressure inside the film, the condition for stability is $\frac{\partial P_{\mathrm{f}}}{\partial e}>0$ [12] giving :

$$
e_{0}=3^{1 / 4} \sqrt{a b} \quad\left(e_{0} \simeq 700 \AA\right) .
$$

For $e_{\mathrm{c}}<e<e_{0}$, films are metastable. 


\section{Building of the meniscus.}

At $t=0$, the fiber is put into contact with a reservoir of liquid (Fig. 4a). We shall focus in this part on the the meniscus (Fig. 4b). The analysis applies for both horizontal and vertical spreading since the lengths involved are much smaller than the capillary length $\kappa^{-1}$.

The liquid meets the solid with a dynamic contact angle $\theta_{d}$ which obeys Tanner's law [13] :

$$
\theta_{\mathrm{d}}^{3} \propto \frac{U}{V^{*}}
$$

where $U$ is the average velocity of the macroscopic contact line located at $h=h_{\mathrm{d}}$ $\left(U=\frac{\mathrm{d} h_{\mathrm{d}}}{\mathrm{d} t}\right)$ and $V^{*}=\frac{\gamma}{\eta}$ is a velocity characteristic of the liquid ( $\eta$ is the liquid viscosity) $\left(V^{*}=2000 \mathrm{~cm} \cdot \mathrm{s}^{-1}\right)$.

We suppose that pressures in the liquid equilibrate quickly so that the profile is given at any time by the profile (20) with angle $\theta_{d}$ :

$$
\cosh \left(\frac{h_{\mathrm{d}}-h_{0}}{b \cos \theta_{\mathrm{d}}}\right)=\frac{1}{\cos \theta_{\mathrm{d}}} .
$$

Near equilibrium $\left|h_{0}-h_{\mathrm{d}}\right| \leqslant b, \theta_{\mathrm{d}} \ll 1$ and (28) becomes :

$$
h_{0}-h_{\mathrm{d}}=b \theta_{\mathrm{d}}
$$

Combining (27) and (29), we obtain :

$$
\frac{\mathrm{d} \theta_{\mathrm{d}}}{\mathrm{d} t} \propto-\frac{V^{*}}{b} \theta_{\mathrm{d}}^{3}
$$

The asymptotic law for $\theta_{\mathrm{d}}$ near equilibrium is thus :

$$
\theta_{\mathrm{d}}^{-2} \propto \frac{t}{\tilde{t}} \quad \text { with } \quad \tilde{t}=\frac{b}{2 V^{*}} \quad\left(\tilde{t} \simeq 2.5 \times 10^{-7} \mathrm{~s}\right) .
$$

For $S \geqslant S_{\mathrm{c}}$ (wetting fluids), the characteristic time for the rise of the meniscus is obtained by setting $\theta_{\mathrm{d}} \simeq\left(\frac{a}{b}\right)^{1 / 3}$ (25) which gives

$$
t_{\mathrm{m}}=\tilde{t}\left(\frac{a}{b}\right)^{-2 / 3} \quad\left(t_{\mathrm{m}} \simeq 0.25 \times 10^{-3} \mathrm{~s}\right)
$$

For non wetting liquids, the contact angle will reach its final value $\theta_{\mathrm{e}}$ for $t=\tilde{t} \theta_{\mathrm{e}}^{-2}$ $\left(\tilde{t} \theta_{\mathrm{c}}^{-2} \simeq 10^{-5} \mathrm{~s}\right.$ for $\left.\theta_{\mathrm{e}}=10^{\circ}\right)$.

\section{Dynamics of the microscopic film.}

4.1 BASIC EQUATIONS [14]. - Let us first examine the two basic equations which determine the profile $e(h, t)$ of the film ( $h$ is the distance from the outer of the drop (horizontal spreading) or the height from the reservoir (ascension)). In the lubrication approximation, 


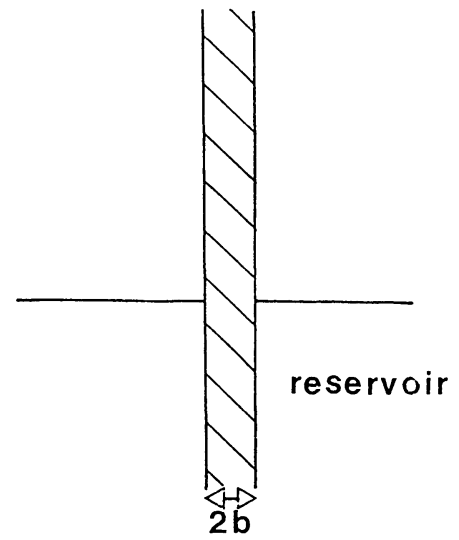

a)
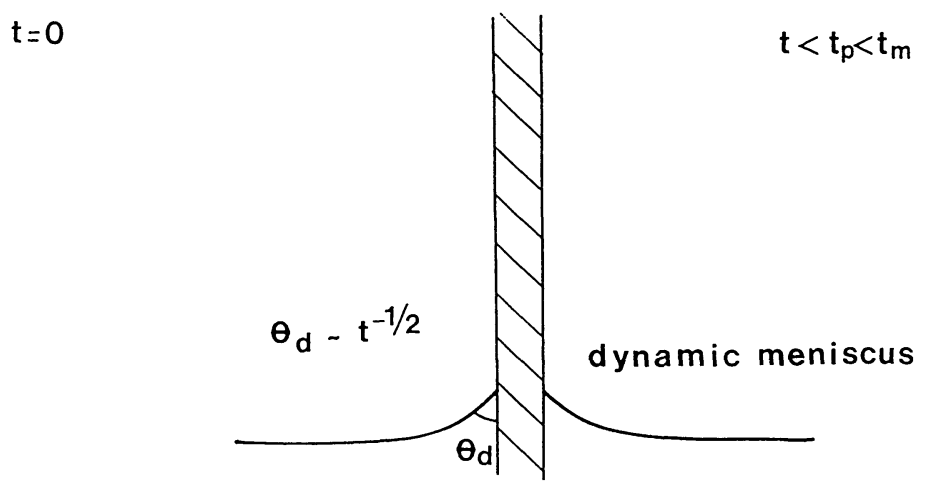

b)

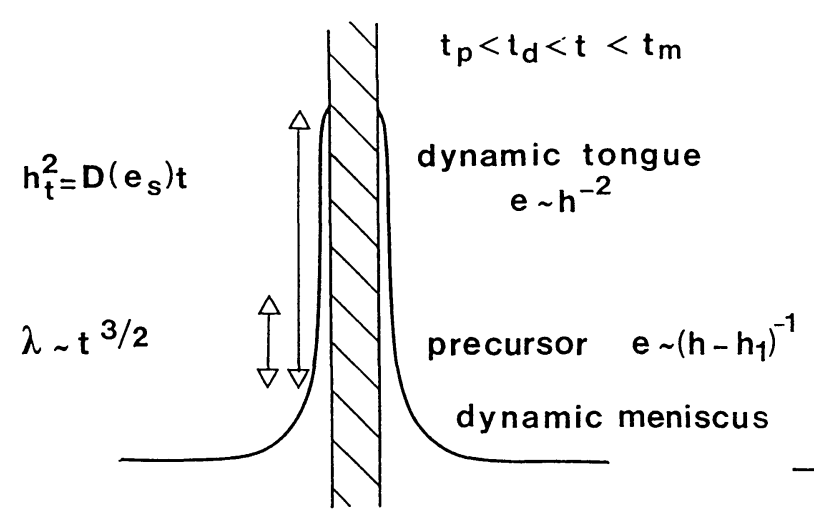

c)

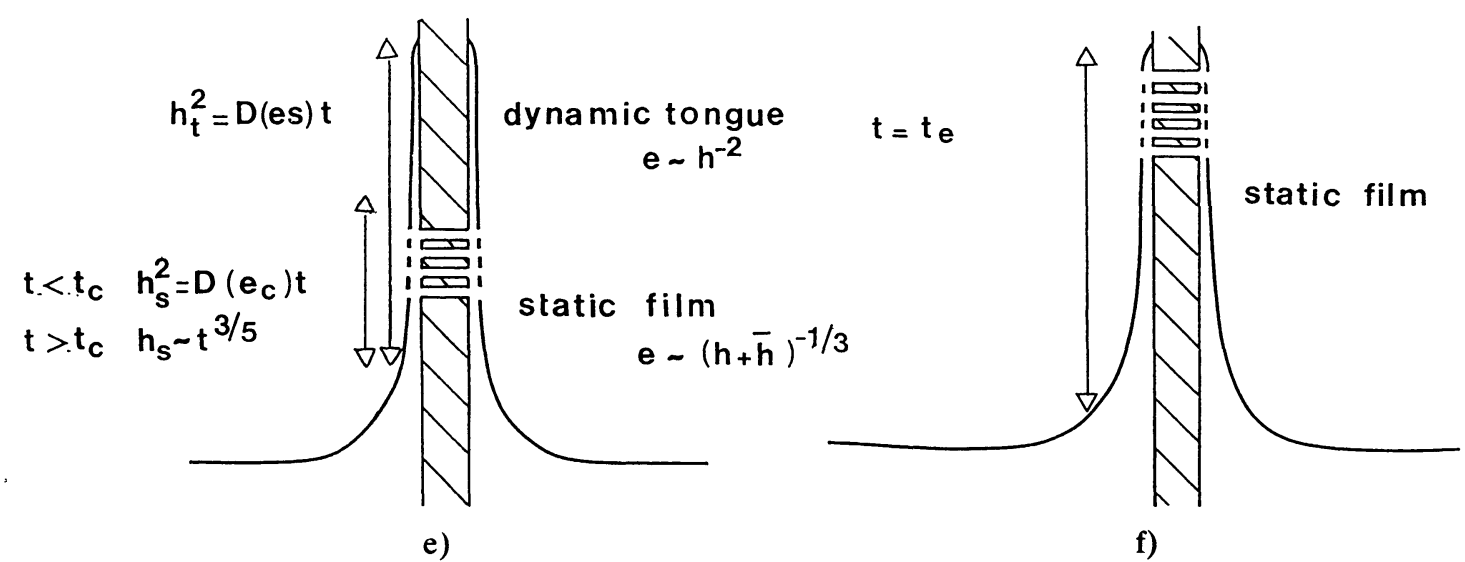

Fig. 4. - Ascension of a liquid on a vertical fiber. a) Initial state $(t=0)$. b) The meniscus develops $\left(t<t_{\mathrm{p}}<t_{\mathrm{m}}\right)$. c) The meniscus now pushes ahead a precursor film and a dynamic tongue diffuses on the fiber $\left(t_{\mathrm{p}}<t_{\mathrm{d}}<t<t_{\mathrm{m}}\right)$. d) The meniscus is fully established : the precursor film has thus disappear. The dynamic tongue still diffuses $\left(t=t_{\mathrm{m}}\right)$. e) The static profile sets up. For $t<t_{\mathrm{c}}$, the boundary between the static film and the diffusive tongue also follows a diffusive motion but for $t>t_{\mathrm{c}}$, it will « accelerate » to meet the tip of the diffusive tongue. f) $t=t_{\mathrm{c}}$ : the boundary between the static and dynamic film has reached the tip of the dynamic film; this is the final equilibrium in figure 3. 
the mean velocity of the liquid front $U(h, t)$ is related to the pressure gradient $-\frac{\partial P}{\partial h}$ (assuming Poiseuille flow) by :

$$
3 \eta \frac{U}{e^{2}}=-\frac{\partial P}{\partial h} .
$$

The pressure $P_{\mathrm{f}}$ inside the film is deduced from the free energy $f$ by $P_{\mathrm{f}}=\frac{\partial f / \partial e}{\partial \Omega / \partial e}$ where $\Omega$ is the volume of wetting liquid per unit length along the fiber. We thus obtain :

$$
P_{\mathrm{f}}=\frac{\gamma}{b+e}-\Pi(e)+\rho g h .
$$

For horizontal spreading, $g=0$.

The second equation is the mass conservation :

$$
\frac{\mathrm{d}}{\mathrm{d} h}(e U)+\frac{\partial e}{\partial t}=0
$$

Using these equations, we now present chronologically all the events that should occur during the spreading phenomenon.

4.2 PreCURSOR FILM. - During its ascension along the fiber, the meniscus pushes ahead a microscopic film (the so-called precursor film) which dissipates the energy $S$ [14]. The gradient of the pressure to be used in the lubrication equation (33) reads :

$$
\frac{\partial P}{\partial h} \simeq\left(-\frac{\gamma}{b^{2}}+3 \frac{\gamma a^{2}}{e^{4}}\right) \frac{\partial e}{\partial h}+\rho g .
$$

Since the height of the meniscus is small compared to the capillary length $\kappa^{-1}$, the last term can be neglected. On the other hand, the first term, which is the gradient of the Laplace pressure, can also be ignored because the thickness of the precursor film is smaller than the threshold value $e_{0}$ for the Rayleigh instability to set in. So (32) reduces to :

$$
\frac{U}{V^{*}}=-\frac{a^{2}}{e^{2}} \frac{\mathrm{d} e}{\mathrm{~d} h},
$$

the solution of which is

$$
e(h, t)=\frac{V^{*}}{U} \frac{a^{2}}{h-h_{1}},
$$

where $h_{1}$ is a time-dependent integration constant which is determined by matching the slope of the above profile with the slope of the macroscopic meniscus given by (28). At the matching point $\left(e_{1}^{*}, h_{1}^{*}\right)$, we get :

$$
\begin{aligned}
& e_{1}^{*}=\frac{a}{\theta_{\mathrm{d}}} \\
& h_{1}^{*}=h_{\mathrm{d}}-\frac{a}{\theta_{\mathrm{d}}^{2}}
\end{aligned}
$$

and the integration constant $h_{1}$ is :

$$
h_{1}=h_{\mathrm{d}}-\frac{2 a}{\theta_{\mathrm{d}}^{2}}=h_{\mathrm{d}}-2\left(\frac{a}{b}\right) V^{*} t
$$


This precursor film will exist only if $e>e_{\mathrm{s}}$ and the length $\lambda$ of this film will be :

$$
\lambda=h\left(e_{\mathrm{s}}\right)-h_{1}=\frac{a^{2}}{e_{\mathrm{s}}}\left(\frac{t}{\tilde{t}}\right)^{3 / 2} \quad\left(\lambda=2.5 \mu \mathrm{m} \text { for } t=t_{\mathrm{m}}\right)
$$

where $\tilde{t}$ has been introduced in the previous section.

This film will appear as soon as $e_{1}^{*}>e_{\mathrm{s}}$, i.e. at a time $t_{\mathrm{p}}$ given by :

$$
t_{\mathrm{p}}=\tilde{t}\left(\frac{e_{\mathrm{s}}}{a}\right)^{2} \quad\left(t_{\mathrm{p}} \simeq 4 \times 10^{-6} \mathrm{~s}\right) \text {. }
$$

4.3 Diffusive TONGUE : DEATH OF THE PRECURSOR FILM (Fig. 4c). - The precursor film is preceded by a diffusive film which is thinner and whose profile is given by combining (33) and (35) :

$$
\frac{\partial e}{\partial t}=\frac{\partial}{\partial h}\left(\frac{\gamma a^{2}}{\eta e} \frac{\partial e}{\partial h}\right)
$$

This film spreads ahead of the meniscus and its precursor film ; the driving force for this film is the disjoining pressure which "pumps» liquid out from the reservoir. The reasons for neglecting the Laplace and gravitational pressures are the same ones as exposed in the last section. A solution of (43) reads :

$$
e(h, t)=\frac{2 a^{2}}{h^{2}} V^{*} t
$$

The cross-over between the precursor film and this diffusive profile is derived by matching (38) and (44) at the point $\left(e_{2}^{*}, h_{2}^{*}\right)$ :

$$
\begin{aligned}
& e_{2}^{*}=16\left(\frac{a}{b}\right)^{2} \frac{V^{* 2} t^{2}}{b} \\
& h_{2}^{*}=\frac{b^{3 / 2}}{2 \sqrt{2 V^{*}}} t^{-1 / 2}
\end{aligned}
$$

The diffusive profile will appear as soon as $e_{2}^{*}>e_{\mathrm{s}}$, i.e. at the time $t_{\mathrm{d}}$ given by :

$$
t_{\mathrm{d}}=\frac{1}{2}\left(\frac{a}{e_{\mathrm{s}}}\right)^{-1 / 2}\left(\frac{a}{b}\right)^{-1 / 2} \tilde{t} \quad\left(t_{\mathrm{d}} \simeq 5 \times 10^{-5} \mathrm{~s}\right) .
$$

The viscous dissipation associated with the building of the meniscus occurs in the precursor film, so the film will die away with the establishment of the static meniscus, i.e. at time $t_{\mathrm{m}}$ given by (32). At this moment, a static film (i.e. the final profile) should start to develop.

\subsection{Development of THE STAtic Film (Fig. 4e).}

4.4.1 Horizontal spreading. - The situation is pictured in figure 5. The equation for the profile is still (43), but now, we need to impose a new boundary condition :

$$
e(h=0, t)=e_{\mathrm{c}} .
$$




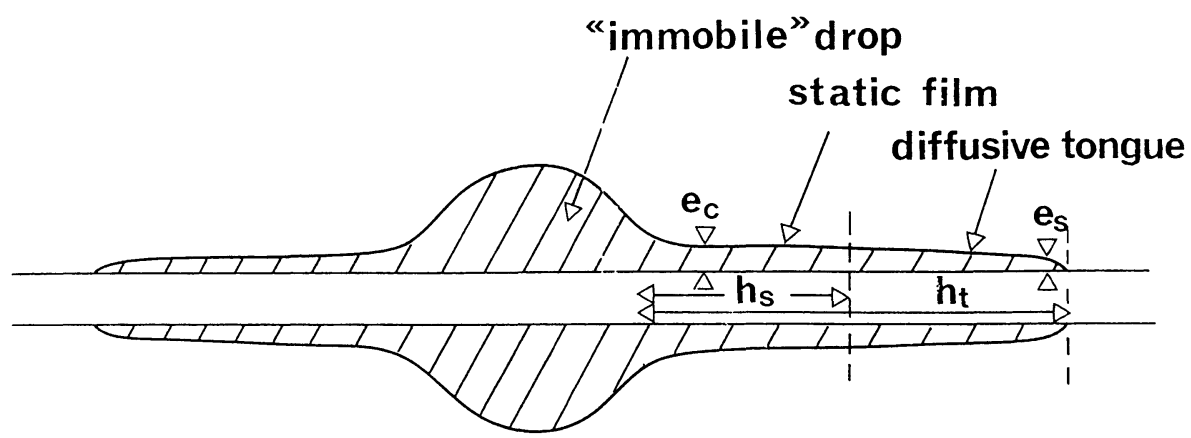

Fig. 5. - Horizontal spreading : a static film of thickness $e_{\mathrm{c}}$ meets a dynamic (and diffusive) tongue of length $h_{\mathrm{t}}$ at $h=h_{\mathrm{s}}$ (which also follows a diffusion law but with a smaller diffusion coefficient).

We re-define the origin of height $h$ by this boundary condition. The solution of such a differential equation is :

$$
\begin{array}{lll}
e(h, t)=2 V^{*} a^{2} \frac{1}{h^{2}} t & \text { for } & h>h_{\mathrm{s}} \\
e(h, t)=e_{\mathrm{c}} & \text { for } & h<h_{\mathrm{s}} .
\end{array}
$$

The static film ends for $h=h_{\mathrm{s}}$ :

$$
h_{\mathrm{s}}^{2}=2 \frac{V^{*} a^{2}}{e_{\mathrm{c}}} t
$$

while the diffusive tongue ends for $h=h_{\mathrm{t}}, e=e_{\mathrm{s}}$ :

$$
h_{\mathrm{t}}^{2}=2 \frac{V^{*} a^{2}}{e_{\mathrm{s}}} t
$$

We have thus shown that the static sheath of thickness $e_{\mathrm{c}}$ spreads out of the meniscus with a simple diffusion law :

$$
h_{\mathrm{s}}^{2}=D\left(e_{\mathrm{c}}\right) t
$$

with the diffusion coefficient given by :

$$
D\left(e_{\mathrm{c}}\right)=2 a V^{*}\left(\frac{a}{b}\right)^{1 / 3} \quad\left(D\left(e_{\mathrm{c}}\right) \simeq 4 \times 10^{-6} \mathrm{~cm}^{2} \mathrm{~s}^{-1}\right) .
$$

The static film will take the place of the dynamic tongue whose tip also follows a diffusion equation but with a larger diffusion coefficient :

$$
D\left(e_{\mathrm{s}}\right)=2 \frac{a^{2}}{e_{\mathrm{s}}} V^{*} \quad\left(D\left(e_{\mathrm{s}}\right) \simeq 3 \times 10^{-5} \mathrm{~cm}^{2} \mathrm{~s}^{-1}\right) .
$$

This analysis applies for a large reservoir or a large drop with negligible Laplace inner pressure. For small drops, the Laplace pressure will slightly thicken the microscopic film and accelerate the spreading.

In our experiments [7], we have also found a diffusive progression of the film but with a 
diffusion coefficient that is $10^{4}$ larger ! The reason was that, as is very often, there were axial grooves along the fibers which conveyed the liquid by capillarity.

4.4.2 Upward creep : gravity effects. - If we include the effects of gravity, the differential equation which describes the profile becomes :

$$
\frac{\partial e}{\partial t}=\frac{\rho g}{\eta} e^{2} \frac{\partial e}{\partial h}+\frac{\partial}{\partial h}\left(\frac{\gamma a^{2}}{\eta e} \frac{\partial e}{\partial h}\right) .
$$

This equation has no analytical solution and we are thus led to look for a self-similar solution [15] in the form of :

$$
e(h, t)=e_{\ell}(t) \varphi\left(\frac{h}{h_{\ell}(t)}\right)
$$

(54) which leads to :

$$
\begin{aligned}
& e_{\ell}(t)=\kappa^{-4 / 5} a^{2 / 5}\left(V^{*} t\right)^{-1 / 5} \\
& h_{\ell}(t)=\kappa^{2 / 5} a^{4 / 5}\left(V^{*} t\right)^{3 / 5}
\end{aligned}
$$

where the function $\varphi$ has to meet the dimensionless differential equation :

$$
\frac{\mathrm{d} \varphi}{\mathrm{d} u}\left(\varphi^{2}+\frac{3}{5} u\right)+\frac{\mathrm{d}}{\mathrm{d} u}\left(\frac{1}{\varphi} \frac{\mathrm{d} \varphi}{\mathrm{d} u}\right)+\frac{\varphi}{5}=0
$$

with $u=\frac{h}{h_{\ell}(t)}$. The former analysis for the case of horizontal spreading leads us to expect two regimes :

a) at small height ( $u$ small) the static film is already established: viscous terms are negligible and (57) becomes

$$
\varphi^{2} \frac{\mathrm{d} \varphi}{\mathrm{d} u}+\frac{\mathrm{d}}{\mathrm{d} u}\left(\frac{1}{\varphi} \frac{\mathrm{d} \varphi}{\mathrm{d} u}\right)=0
$$

with the boundary condition : $\left(h=0, e=e_{\mathrm{c}}\right)$. Equation (58) can be integrated to give :

$$
\varphi(u)=(u+\bar{u})^{-1 / 3}
$$

with

$$
\bar{u}=\left(\frac{e_{\ell}(t)}{e_{\mathrm{c}}}\right)^{3}=\frac{\bar{h}}{h_{\ell}(t)} \quad \text { and } \quad \bar{h}=\frac{\kappa^{-2}}{b}
$$

Equation (59) is of course identical to equation (22): it describes the static profile.

b) at large height, a similar analysis leads to the dynamical profile given by (44) (this film is thinner and thus not subject to gravity):

$$
\varphi(u)=\frac{2}{u^{2}}
$$

The cross-over between the static film and the diffusive tongue depends on $\bar{u}$ : the profiles (59) and (60) match for :

$$
(u+\bar{u})^{-1 / 3}=2 u^{-2}
$$


We call $t_{\mathrm{c}}$ the time defined by $\bar{u}=1$ :

$$
t_{\mathrm{c}}=\frac{1}{V^{*}} \kappa^{-4} a^{-4 / 3} b^{-5 / 3} \quad\left(t_{\mathrm{c}} \simeq 1 \text { year }\right) .
$$

This time defines the timescale and height for which gravity becomes important. Up to $\bar{h}=\frac{\kappa^{-2}}{b}(\bar{h}=10 \mathrm{~cm})$, gravity is negligible : for short time $\left(t<t_{\mathrm{c}}\right.$, i.e. $\left.u<\bar{u}\right)$, the matching point is given by (49) ( $g=0$ or horizontal spreading).

When $t>t_{\mathrm{c}}$, the solution of (61) is $u \simeq 1$, i.e. :

$$
h_{\mathrm{s}}(t)=h_{\ell}(t)=\kappa^{2 / 5} a^{4 / 5}\left(V^{*} t\right)^{3 / 5} .
$$

The static film no longer follows a diffusion law and creeps faster than the diffusive dynamic film. Finally, both films meet for $h=\bar{H}(23)$, the final height of the static film. This occurs for $t=t_{\mathrm{c}}$ :

$$
t_{\mathrm{e}}=\frac{\bar{H}^{2}}{D\left(e_{\mathrm{s}}\right)}
$$

(recall that the dynamic film always spreads with a diffusion law). The final equilibrium, therefore, will be reached at very long time ( $t_{\mathrm{e}} \simeq \mathrm{a}$ few thousands of years) but remember that we are studying flow in an annular pipe of thickness only a few $\AA$, and moreover that this flow obeys a diffusion law. Obviously, only the first stages of the spreading can be experimentally observed.

\section{Conclusion.}

This theory is very similar to that of Joanny and de Gennes for planar surfaces [15]. For very thin films (here the precursor film and the dynamic tongue), curvature effects due to the fiber can be ignored, and so the results are the same. For thicker films (meniscus and microscopic static films), these curvature effects have to be considered.

The meniscus on a fiber, contrarily to the case of planar surfaces, has zero total curvature and climbs up to height of order $b$ (fiber radius), small compared to $\kappa^{-1}$ (height of the meniscus for a vertical planar surface). Hence, the building of the meniscus is quite quicker (by a factor of $\kappa^{-1} / b$ of order 100 ). It could be experimentally studied by using very viscous liquids.

The thickness of the microscopic static film is also related to the radius $b$, in both situations (i.e. vertical or horizontal fibers). As for planar surfaces, the spreading of microscopic films presents an interesting cascade of regimes, but the timescales are different here. Another special feature of fibers has to be emphasized : for a vertical fiber, gravity effects can be ignored for $h$ (height of the tip of the film above the reservoir) smaller than $\kappa^{-2} / b$ (the latter quantity can be order $10 \mathrm{~cm}$ ). Since the progression of the microscopic film is very slow, the no-gravity description should be enough for many practical situations.

\section{Acknowledgments.}

David Quéré thanks ATOCHEM for financial support. 


\section{References}

[1] Lord Rayleigh, Proc. London Math. Soc. 10 (1879) 4.

[2] Brochard F., J. Chem. Phys. 84 (1984) 4664.

[3] di Meglio J. M., Quéré D. and Brochard-Wyart F., C.R. Acad. Sci. Sér. II 309 (1989) 19.

[4] Carroll B. J., J. Coll. Int. Sci. 97 (1984) 195.

[5] di Meglio J. M., C.R. Acad. Sci. Sér. II 303 (1986) 437.

[6] Quéré D., Di Meglio J. M. and Brochard-Wyart F., Europhys. Lett. 10 (1989) 335.

[7] Quéré D., di Meglio J. M. and Brochard-Wyart F., Revue Phys. Appl. 23 (1988) 1023.

[8] For a review on wetting phenomena, see :

De Gennes P. G., Rev. Mod. Phys. 57 (1985) 828 ;

JoAnNy J. F., Thèse de Doctorat d'Etat, Université Paris VI (1985).

[9] Derjaguin B. V. and Churaev, N. V., J. Coll. Int. Sci. 49 (1974) 249.

[10] Joanny J. F. and de Gennes P. G., C.R. Acad. Sci. Sér. II 299 (1984) 279.

[11] Brochard-Wyart F., C.R. Acad. Sci. II 303 (1986) 1077.

[12] Starov V. M. and Churaev N. V., Kolloidnyi Zhurnal 40 (1978) 909.

[13] Hoffman R., J. Coll. Int. Sci. 50 (1975) 228.

[14] De Gennes P. G., C.R. Acad. Sci. Sér. II 298 (1984) 439.

[15] Joanny J. F. and De Gennes P. G., J. Phys. France 47 (1986) 121. 